\title{
The innovation and clinical application of ischemia-free organ transplantation
}

\author{
Zhiyong Guo ${ }^{1,2,3}$, Tao Luo ${ }^{1,2,3}$, Qiang Zhao ${ }^{1,2,3}$, and Xiaoshun $\mathrm{He}^{1,2,3, *}$ \\ 1 Organ Transplant Center, The First Affiliated Hospital, Sun Yat-sen University, Guangzhou 510080, China \\ 2 Guangdong Provincial Key Laboratory of Organ Donation and Transplant Immunology, Guangzhou 510080, China \\ ${ }^{3}$ Guangdong Provincial International Cooperation Base of Science and Technology, Guangzhou 510080, China
}

Received 24 August 2021, Accepted 13 October 2021, Published online 1 December 2021

\begin{abstract}
The incidence rate of liver cancer is increasing year by year globally. Liver transplantation has become one of the therapeutic methods for patients with liver cancer. In the past, ischemia-reperfusion injury (IRI) was unavoidable in liver transplantation, compromising recipient and graft survival. At the same time, the imbalance between the supply and demand of organs limits the use of transplantation in patients with liver cancer. Recently, advances have been made in machine perfusion techniques to reduce graft IRI. However, none of the techniques can completely abrogate graft IRI. In 2017, the concept of ischemia-free organ transplantation (IFOT) was proposed, and our group conducted the first case of ischemia-free liver transplantation (IFLT). We then extended the concept of IFOT to kidney transplantation in 2018 and to heart transplantation in 2021. Here, we review the history, strengths, and weaknesses, and the future direction of IFOT, particularly in patients with liver cancers.
\end{abstract}

Key words: Liver cancer, Ischemia-reperfusion injury, Ischemia-free organ transplantation, Ischemia-free liver transplantation, Extended criteria donor.

\section{Introduction}

In the past 60 years, cancer has become the first or second leading cause of premature death in 134 of 183 countries and the third or fourth primary cause in the rest of the countries according to the WHO's Global Health Estimates [1]. Specifically, in the countries with high levels of the Human Development Index (like China and Brazil), cancer is the second leading cause of premature death [1].

Among the most common cancers and a most common cause of cancer death worldwide, liver cancer ranked sixth and fourth in 2018 [2]. There were 953,000 incident cases of liver cancer and 819,000 deaths globally in 2017 , contributing to 20.8 million disability-adjusted life years (DALYs). Of the $35 \%$ increase rate between 2007 and 2017, 17\% can be explained by population aging, $13 \%$ by population growth, and $6 \%$ by increasing age-specific incidence rates [3].

In China, the incidence of liver cancer ranked third among all cancers, and the mortality rate ranked second in 2015. The contribution of hepatitis B virus, hepatitis C virus, alcohol, and other causes on absolute liver cancer deaths in china were $41 \%, 8 \%, 33 \%$, and $18 \%$, respectively [4]. Surgical resection is the mainstay of treatment in well-selected candidates with primary or secondary liver tumors [5-8]. According to the

\footnotetext{
*Corresponding author: gdtrc@163.com
}

European Association for the Study of the Liver (EASL) Clinical Practice Guidelines for Management of hepatocellular carcinoma in 2018, in patients with early tumors, surgical resection and orthotopic liver transplantation (OLT) are the first choices on an intention-to-treat perspective [7].

In 1963, Starzl et al. performed the first OLT in humans [9]. OLT has been associated with efficient improvements in the survival of patients with end-stage liver disease [10-13]. The Milan criteria are the benchmark for liver transplantation in patients with liver cancer [14], while the Child-Pugh-Turcotte classification and the model for end-stage liver disease (MELD) score are used to evaluate the patient priority on the waiting list [15]. For patients meeting Milan criteria but not suitable for liver surgical resection, OLT is recommended as the first-line treatment option [7]. Moreover, hepatocellular carcinoma (HCC), as a particular MELD exception, requires extra points to enter the transplant routine, standardized in different countries considering size, number of nodules, alpha-fetoprotein levels, recurrence after tumor downstaging [13].

\section{The history and achievement of ischemia-free organ transplantation (IFOT)}

Ischemia-reperfusion injury (IRI) in organ transplantation is unavoidable, leading to early allograft dysfunction (EAD), 
primary nonfunction (PNF), immune rejection response, and the other complication [16-18]. IRI damages all parenchymal cells in the liver, including hepatocytes, sinusoidal endothelial cells, and biliary epithelial cells [19]. The mechanisms mainly contain reactive oxygen species (ROS) [20], toll-like receptor activation [21], autophagy regulation [22], and hypoxia-inducible factor (HIF) activation [23], which connect parenchymal cells, kupffer cells, T cells, neutrophils, and platelets in the liver to form the network of IRI pathogenesis.

Various mechanical perfusion techniques have been clinically proven to reduce IRI and improve transplant outcomes, including hypothermic machine perfusion (HMP) [24], hypothermic oxygenated perfusion (HOPE) [25], subnormothermic machine perfusion (SNP) [26], controlled oxygenated rewarming (COR) [27], normothermic machine perfusion (NMP) [28]. However, these techniques alone cannot eliminate graft IRI because the organs still suffer ischemia during procurement and implantation.

To completely avoid graft IRI, the transplant team of The First Affiliated Hospital of Sun Yat-sen University in China proposed a unique solution, the IFOT procedure, during which the donor organs are procured, preserved, and implanted without interruption of blood supply by using NMP. In 2017, $\mathrm{He}$ and his colleagues performed the first ischemia-free liver transplantation (IFLT) in humans [29]. The perfusion inflow is established by cannulization of the portal vein and gastroduodenal/splenic artery. The perfusion outflow is established by cannulization of the inferior vena cava. The cannulas are connected with the perfusion lines or organ reservoir of an NMP device. After the in-situ NMP is set up, the liver is procured and moved to the organ reservoir of the NMP device for continuous ex-situ NMP (Video 1). After the hepatectomy of the diseased liver is completed, the donor's liver is moved to the recipient's abdominal cavity and implanted under in-situ NMP. Importantly, the donor's liver was with 85-90\% macrovesicular hepatosteatosis, which is usually discarded in the majority of transplant centers all over the world. Moreover, the pathological studies of the liver biopsy samples suggest that graft IRI is largely avoided. Therefore, the first case confirmed the feasibility and efficacy of the concept of IFOT.

In 2018, our group expanded the IFOT concept to kidney transplantation and conducted the first case of ischemia-free kidney transplantation (IFKT) in humans [30]. We recently moved forward to the ischemia-free beating heart transplantation (IFBHT) on June 26th, 2021. A donor heart with an ischemic time over $6 \mathrm{~h}$ is considered a marginal one and often discarded. Prolonged ischemic time of donor's heart is significantly associated with inferior graft survival and increased allograft rejection [31]. During IFBHT, the donor's heart is transplanted under an ischemia-free, beating, normothermic, oxygenated perfused state. Therefore, IFBHT provides a brand new approach to increase donor heart availability and improve transplant outcomes.

One of the advantages of IFOT is that graft viability can be easily assessed during the procedure. We analyzed the parameters of liver perfusion in 28 IFLT cases to identify the correlation between perfusion parameters, such as vascular flow/pressure, blood gas analysis of the perfusate and bile, liver function tests of the perfusate, and the post-transplant outcomes [32].

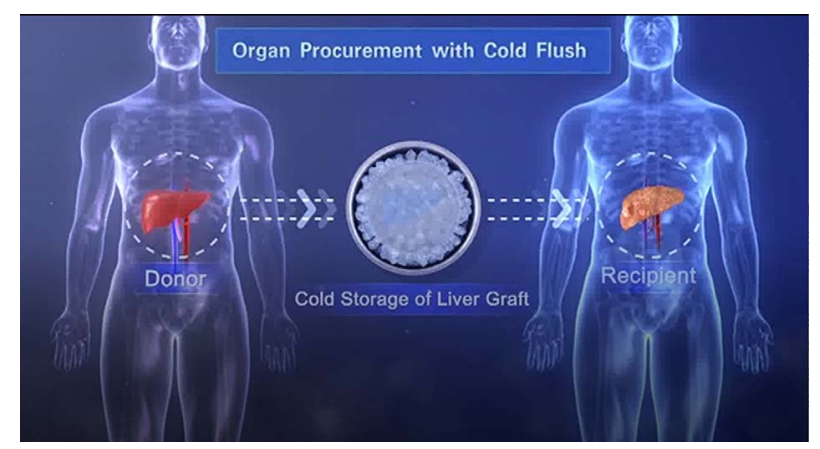

Video 1. Conventional cold storage (CCS) has been the standard conventional preservation technique in liver transplantation, with the unavoidable graft damage in the reperfusion stage. This video shows the ischemia-free liver transplantation (IFLT), during which the donor organs are procured, preserved and implanted without interruption of blood supply by using normothermic machine perfusion (NMP). https://vcm.edpsciences.org/10.1051/vcm/2021005\#V1.

We established an objective criterion of transplant ability in marginal donor livers based on these parameters. Importantly, because there is no ischemia before implantation, the graft function is stable, which makes the assessment much easier than other approaches.

In 2021, our group published the results of the first prospective, single-center, non-randomized controlled trial [33]. The study showed that IFLT can foster liver graft recovery and reduce the incidence of IRI-related complications. Importantly, by using the new technique, the livers from extended criteria donors (ECD) yielded a faster recovery than those from standard criteria donors (SCD) undergoing conventional transplant techniques. The pathological, transcriptome, proteome and metabolome studies of the liver biopsy samples demonstrated that graft IRI is largely avoided by using IFLT [34].

\section{The application of ischemia-free liver transplantation in liver cancer patients}

Liver cancer is one of the major indications for liver transplantation in China. From January 1st, 2015, to December 31st, $2019,52 \%$ of patients receiving liver transplantation had liver malignancies in our center. Studies have shown that profound graft IRI is associated with the recurrence of liver cancer [35-37]. Since graft IRI is largely abrogated in IFLT, the incidence of post-transplant cancer recurrence might be lower in IFLT versus conventional liver transplantation. Interestingly, the recipient of the first case of IFLT was a 51-year-old man with decompensated liver cirrhosis and hepatocellular carcinoma (HCC). We are collecting data concerning the cancer prognosis in the new and conventional transplant procedure. The preliminary data suggest that IFLT can reduce cancer recurrence (unpublished data).

\section{The strengths and weaknesses of IFOT}

Robert et al. from the University of Groningen highly recognizes our team's pioneering work in the field of organ 
transplantation and made a comment that "the team led by Dr. $\mathrm{He}$ is to be commended for this great achievement, which we consider a milestone in the history of organ transplantation" [38]. Quintini et al. from Cleveland Clinic commented that "to achieve what researchers worldwide had pursued for decades: the abrogation of IRI in transplantation" [39]. Jayant et al. from Imperial London College considered our technique "realized the dream of ischemia-free organ transplantation" [40]. Kim et al. from Medical College of Wisconsin commented that "The ischemia-free organ transplantation (IFOT) achieved complete avoidance of IRI" [41].

On the other hand, there are limitations of the novel technique at the current stage. Firstly, a transportable NMP device is required to make distant procurement of donor organs possible. Secondly, the surgical techniques need to be simplified. Thirdly, a multicenter trial is pending to confirm the clinical benefits of IFOT.

\section{The future direction of IFOT}

There are debates on whether NMP can repair damaged organs or simply reduce the cold ischemic injury. Every year, a large number of marginal organs are discarded because of pre-existing damages. The maximum benefits of IFOT depend on the repairing abilities of NMP. Therefore, the major direction of IFOT includes (1) elongating the NMP duration [42], (2) adding defatting agents to the perfusate to reduce liver steatosis [43, 44], stem cells or organoids [45], anti-inflammation agents [46, 47], and miRNA therapy in the NMP perfusate [48]. More importantly, IFOT opens the window of organ-based medicine [49]. Based on the ex-situ multiple organ NMP platforms, the diseases of specific human organs can be studied and treated in an isolated state, which might promote the advances of medical science in the near future.

\section{Conflict of interest}

The authors have nothing to disclose.

\section{References}

1. Yoon JU, Byeon GJ, Park JY, et al. Bloodless living donor liver transplantation: risk factors, outcomes, and diagnostic predictors. Medicine (Baltimore) 2018;97(50):e13581.

2. Bray F, Ferlay J, Soerjomataram I, et al. Global cancer statistics 2018: GLOBOCAN estimates of incidence and mortality worldwide for 36 cancers in 185 countries. CA Cancer J Clin 2018;68(6):394-424.

3. Global Burden of Disease Cancer C, Fitzmaurice C, Abate D, et al. Global, regional, and national cancer incidence, mortality, years of life lost, years lived with disability, and disabilityadjusted life-years for 29 cancer groups, 1990 to 2017: a systematic analysis for the global burden of disease study. JAMA Oncol. 2019;5(12):1749-1768.

4. Global Burden of Disease Liver Cancer CAkinyemiju T, Abera S, et al. The burden of primary liver cancer and underlying etiologies from 1990 to 2015 at the global, regional, and national level: results from the Global Burden of Disease Study 2015. JAMA Oncol 2017;3(12):1683-1691.

5. Martel G, Hawel J, Rekman J, et al. Liver resection for noncolorectal, non-carcinoid, non-sarcoma metastases: a multicenter study. PLoS One 2015;10(3):e0120569.

6. Uggeri F, Ronchi PA, Goffredo P, et al. Metastatic liver disease from non-colorectal, non-neuroendocrine, non-sarcoma cancers: a systematic review. World J Surg Oncol. 2015;13:191.

7. European Association for the Study of the Liver. Electronic address eee, European Association for the Study of the L. EASL Clinical Practice Guidelines: Management of hepatocellular carcinoma. J Hepatol 2018;69(1):182-236.

8. Tsilimigras DI, Brodt P, Clavien P-A, et al. Liver metastases. Nat Rev Dis Primers 2021;7(1):27.

9. Starzl TE, Marchioro TL, Vonkaulla KN, et al. Homotransplantation of the liver in humans. Surg Gynecol Obstet 1963;117:659-676.

10. Merion RM, Schaubel DE, Dykstra DM, et al. The survival benefit of liver transplantation. Am J Transplant 2005;5(2): 307-313.

11. Dawwas MF, Gimson AE, Lewsey JD, et al. Survival after liver transplantation in the United Kingdom and Ireland compared with the United States. Gut 2007;56(11):1606-1613.

12. Adam R, Karam V, Delvart V, et al. Evolution of indications and results of liver transplantation in Europe. A report from the European Liver Transplant Registry (ELTR). J Hepatol 2012; 57(3):675-688.

13. European Association for the Study of the Liver. Electronic address EEE. EASL Clinical Practice Guidelines: Liver transplantation. J Hepatol 2016;64(2):433-485.

14. Mazzaferro V, Regalia E, Doci R, et al. Liver transplantation for the treatment of small hepatocellular carcinomas in patients with cirrhosis. New England J Med 1996;334(11):693-699.

15. Wiesner R, Edwards E, Freeman R, et al. Model for end-stage liver disease (MELD) and allocation of donor livers. Gastroenterology 2003;124(1):91-96.

16. de Rougemont O, Dutkowski P, Clavien PA. Biological modulation of liver ischemia-reperfusion injury. Curr Opin Organ Transplant 2010;15(2):183-189.

17. Zhai Y, Petrowsky H, Hong JC, Busuttil RW, KupiecWeglinski JW. Ischaemia-reperfusion injury in liver transplantation - from bench to bedside. Nat Rev Gastroenterol Hepatol 2013;10(2):79-89.

18. Ito T, Naini BV, Markovic D, et al. Ischemia-reperfusion injury and its relationship with early allograft dysfunction in liver transplant patients. Am J Transplant 2020;21(2):614-625.

19. Dar WA, Sullivan E, Bynon JS, et al. Ischaemia reperfusion injury in liver transplantation: Cellular and molecular mechanisms. Liver Int 2019;39(5):788-801.

20. Reiniers MJ, van Golen RF, van Gulik TM, et al. Reactive oxygen and nitrogen species in steatotic hepatocytes: a molecular perspective on the pathophysiology of ischemia-reperfusion injury in the fatty liver. Antioxid Redox Signal 2014;21(7): 1119-1142.

21. Bamboat ZM, Balachandran VP, Ocuin LM, et al. Toll-like receptor 9 inhibition confers protection from liver ischemiareperfusion injury. Hepatology 2010;51(2):621-632.

22. Cursio R, Colosetti P, Gugenheim J. Autophagy and liver ischemia-reperfusion injury. Biomed Res Int 2015;2015:17590. 
23. Yang YY, Lee PC, Huang YT, Lee WP, et al. Involvement of the HIF-1alpha and Wnt/beta-catenin pathways in the protective effects of losartan on fatty liver graft with ischaemia/reperfusion injury. Clin Sci (Lond) 2014;126(2):163-174.

24. van Rijn R, Schurink IJ, de Vries Y, et al. Hypothermic machine perfusion in liver transplantation - a randomized trial. N Engl J Med 2021;384(15):1391-1401.

25. Kron P, Schlegel A, Mancina L, et al. Hypothermic oxygenated perfusion (HOPE) for fatty liver grafts in rats and humans. J Hepatol 2017;S0168-8278(17)32268-7.

26. Bruinsma BG, Yeh H, Ozer Set al. Subnormothermic machine perfusion for ex vivo preservation and recovery of the human liver for transplantation. Am J Transplant 2014;14(6):1400-1409.

27. Hoyer DP, Mathe Z, Gallinat A, et al. Controlled oxygenated rewarming of cold stored livers prior to transplantation: first clinical application of a new concept. Transplantation 2016; 100(1):147-152.

28. Nasralla D, Coussios CC, Mergental H, et al. A randomized trial of normothermic preservation in liver transplantation. Nature 2018;557(7703):50-56.

29. He X, Guo Z, Zhao Q, et al. The first case of ischemia-free organ transplantation in humans: a proof of concept. Am J Transplant 2018;18(3):737-744.

30. He X, Chen G, Zhu Z, et al. The first case of ischemia-free kidney transplantation in humans. Front Med (Lausanne) 2019;6:276.

31. Lund LH, Khush KK, Cherikh WS, et al. The registry of the International Society for Heart and Lung Transplantation: Thirty-fourth Adult Heart Transplantation Report-2017; focus theme: allograft ischemic time. J Heart Lung Transplant 2017;36(10):1037-1046.

32. Zhang Z, Tang Y, Zhao Qet al. Association of perfusion characteristics and posttransplant liver function in ischemia-free liver transplantation. Liver Transpl 2020;26(11):1441-1454.

33. Guo Z, Zhao Q, Huang S, et al. Ischaemia-free liver transplantation in humans: a first-in-human trial. Lancet Reg Health West Pac. 2021;16:100260.

34. Clinical and Translational Medicine. 2021 (in press).

35. Oldani G, Crowe LA, Orci LA, et al. Pre-retrieval reperfusion decreases cancer recurrence after rat ischemic liver graft transplantation. J Hepatol 2014;61(2):278-285.

36. Kornberg A, Witt U, Kornberg J, et al. Treating ischaemiareperfusion injury with prostaglandin E1 reduces the risk of early hepatocellular carcinoma recurrence following liver transplantation. Aliment Pharmacol Ther 2015;42(9):1101-1110.
37. Nagai S, Yoshida A, Facciuto Met al. Ischemia time impacts recurrence of hepatocellular carcinoma after liver transplantation. Hepatology (Baltimore, MD) 2015;61(3):895-904.

38. van Leeuwen OB, Ubbink R, de Meijer VE, et al. The first case of ischemia-free organ transplantation in humans: A proof of concept. Am J Transplant 2018;18(8):2091.

39. Quintini C, Diago Uso T, Liu Q. Ischemia-free liver transplantation: Will the diamond with a flaw replace the pebble without? Liver Transpl 2020;26(11):1391-1392.

40. Jayant K, Reccia I, Shapiro AMJ. Normothermic ex-vivo liver perfusion: Where do we stand and where to reach? Expert Rev Gastroenterol Hepatol 2018;12(10):1045-1058.

41. Kim J, Zimmerman MA, Hong JC. Emerging innovations in liver preservation and resuscitation. Transplant Proc 2018;50(8): 2308-2316.

42. Mueller M, Hefti M, Eshmuminov D, et al. Long-term normothermic machine preservation of partial livers: first experience with 21 human hemi-livers. Ann Surg 2021;274(5):836-842.

43. Liu Q, Berendsen T, Izamis ML, et al. Perfusion defatting at subnormothermic temperatures in steatotic rat livers. Transplant Proc 2013;45(9):3209-3213.

44. Boteon YL, Stephenson BTF, Neil DAH, et al. Lipid metabolism and functional assessment of discarded human livers with steatosis undergoing 24 hours of normothermic machine perfusion. Liver Transpl 2018;24(5):708-709.

45. Verstegen MMA, Mezzanotte L, Ridwan RY, et al. First report on ex vivo delivery of paracrine active human mesenchymal stromal cells to liver grafts during machine perfusion. Transplantation 2020;104(1):e5-e7.

46. Chiang $\mathrm{CH}, \mathrm{Wu} \mathrm{CP}$, Perng WC, et al. Use of anti-(tumour necrosis factor-alpha) antibody or 3-deaza-adenosine as additives to promote protection by University of Wisconsin solution in ischaemia/reperfusion injury. Clin Sci (Lond) 2000;99 (3):215-222.

47. Donckier V, Troisi R, Le Moine A, et al. Early immunosuppression withdrawal after living donor liver transplantation and donor stem cell infusion. Liver Transpl 2006;12(10):1523-1528.

48. Nassar A, Liu Q, Farias K, et al. Role of vasodilation during normothermic machine perfusion of DCD porcine livers. Int $\mathbf{J}$ Artif Organs 2014;37(2):165-172.

49. Zhao Q, Nie Y, Guo Z, et al. The future of organ-oriented research and treatment. Hepatobiliary Surg Nutr 2019;8(5): 502-505.

Cite this article as: Guo Z, Luo T, Zhao Q \& He X (2021) The innovation and clinical application of ischemia-free organ transplantation. Visualized Cancer Medicine. 2021; 2, 6 